\section{microRNA miR-14 acts to modulate a positive autoregulatory loop controlling steroid hormone signaling in Drosophila}

\author{
Jishy Varghese ${ }^{1}$ and Stephen M. Cohen ${ }^{1,2}$ \\ European Molecular Biology Laboratory, \\ 69117 Heidelberg, Germany
}

The insect steroid hormone Ecdysone and its receptor play important roles during development and metamorphosis and regulate adult physiology and life span. Ecdysone signaling, via the Ecdysone receptor (EcR), has been proposed to act in a positive autoregulatory loop to increase EcR levels and sensitize the animal to ecdysone pulses. Here we present evidence that this involves EcRdependent transcription of the $E c R$ gene, and that the microRNA miR-14 modulates this loop by limiting expression of its target EcR. Ecdysone signaling, via EcR, down-regulates miR-14. This alleviates miR-14-mediated repression of EcR and amplifies the response. Failure to limit EcR levels is responsible for the many of the defects observed in miR-14 mutants. miR-14 plays a key role in modulating the positive autoregulatory loop by which Ecdysone sensitizes its own signaling pathway.

Supplemental material is available at http://www.genesdev.org.

Received May 5, 2007; revised version accepted July 23, 2007.

MicroRNAs (miRNAs) are small noncoding RNAs that serve as post-transcriptional regulators of gene expression (for reviews, see Carthew 2006; Kloosterman and Plasterk 2006; Massirer and Pasquinelli 2006; Bushati and Cohen 2007). Animal miRNAs pair to complementary sequences, typically in the 3' untranslated regions (UTRs) of target RNAs to repress translation and may also destabilize the target RNAs (for review, see Valencia-Sanchez et al. 2006). Animal miRNAs were first encountered as genes affecting developmental timing in Caenorhabditis elegans (Lee et al. 1993; Wightman et al. 1993; Pasquinelli et al. 2000; Reinhart et al. 2000). The lin-4 and let-7 miRNAs regulate expression of transcription factors that control the progression between larval stages in the nematode (Olsen and Ambros 1999; Abbott et al. 2005; Grosshans et al. 2005; Li et al. 2005).

In Drosophila, the steroid hormone Ecdysone and its receptor EcR play a key role in control of developmental timing. Pulses of Ecdysone release from the ring gland

[Keywords: Ecdysone; autorgulatory feedback loop; microRNA; steroid hormone]

${ }^{1}$ Present address: Temasek Life Sciences Laboratory and Department of Biological Sciences, 1 Research Link, The National University of Singapore, Singapore 117604.

${ }^{2}$ Corresponding author.

E-MAIL cohen@tll.org.sg; FAX 65-6872-7089.

Article published online ahead of print. Article and publication date are online at http://www.genesdev.org/cgi/doi/10.1101/gad.439807. induce the major developmental transitions: from embryo to larva, the larval molts, from larva to pupa, and from pupa to adult (for review, see Thummel 2001). The binding of Ecdysone to the EcR complex triggers a complex hierarchy of gene activation that controls the physiological and morphological changes involved in metamorphosis (for review, see Thummel 1996). An important feature of this process is its all-or-none character, and previous work has identified a positive autoregulatory component to the ecdysone response, as Ecdysone signaling increases the level of its receptor (Koelle et al. 1991; Karim and Thummel 1992). In this report we examine the mechanistic basis for autoregulation of this ecdysone response and present evidence for both a transcriptional component and for an indirect modulatory role of the miRNA miR-14.

\section{Results and Discussion}

The Drosophila EcR gene was identified computationally as a possible target for $m i R-14$ (Enright et al. 2003; Stark et al. 2003). EcR produces five mRNAs that encode three different proteins-EcR-A, EcR-B1 and EcR-B2but all transcripts contain the same 1.8-Kb 3' UTR. The EcR 3' UTR contains three predicted target sites for $m i R$ 14 (Fig. 1A), which are conserved in distantly related Drosophila species (Supplementary Fig. S1). To test whether miR-14 can regulate the EcR 3' UTR, S2 cells were transfected to express a luciferase reporter containing either a control 3' UTR or that of EcR. The cells were also transfected to express miR-14. miR-14 expression reduced activity of the luciferase reporter containing the EcR 3' UTR by $\sim 40 \%$, but showed a reduced ability to regulate a comparable construct in which the seed regions of the three conserved miR-14 target sites had been mutated (Fig. 1B). Similar results were obtained in transgenic Drosophila, where Gal4-directed expression of UAS-miR-14 transgene reduced expression of a GFP reporter transgene containing the $3^{\prime}$ UTR of EcR in the wing imaginal disc (Fig. 1C). Gal4-directed expression of miR-14 also reduced the level of the endogenous EcR protein in the wing disc (Fig. 1D). Thus miR-14 is able to regulate EcR in vivo, at least when overexpressed.

To assess the ability of endogenous miR-14 to regulate $E c R$ expression, we examined EcR protein levels in extracts from control pupae and pupae lacking miR-14. EcR protein was more abundant in the miR-14 mutant samples (Fig. 2A). Likewise, GFP levels increased when the GFP reporter transgene containing the EcR 3' UTR was examined in the miR-14 mutant (Fig. 2B). Taken together with the results in Figure 1, B and C, these findings provide evidence that miR-14 acts directly, via the 3'UTR of the EcR mRNA, to control EcR expression.

To ask if the elevated level of EcR protein in the $m i R$ 14 mutant confers elevated Ecdysone-responsive gene expression, we used a reporter construct in which seven copies of the hsp27 Ecdysone response element direct lac $Z$ expression, providing a direct readout for Ecdysone signaling (White et al. 1997). The level of $\beta$-Galactosidase protein expressed by the Ec-RE:lacZ reporter was higher in the miR-14 mutant background (Fig. 2C). Next we measured the mRNA levels for three known EcR target genes-E74, E93, and Fbp1-at 5 and $24 \mathrm{~h}$ after puparium formation $(\mathrm{APF})$; expression of each target was 
A

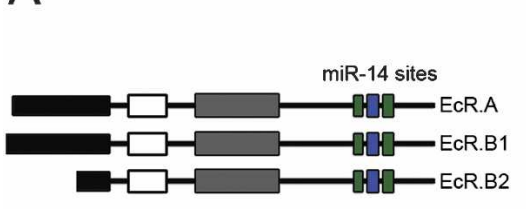

B

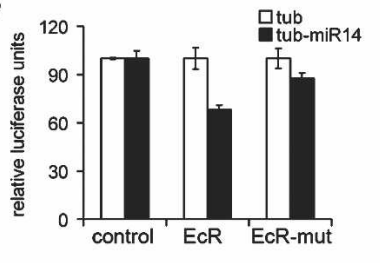

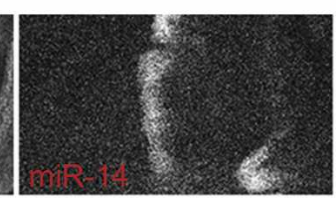
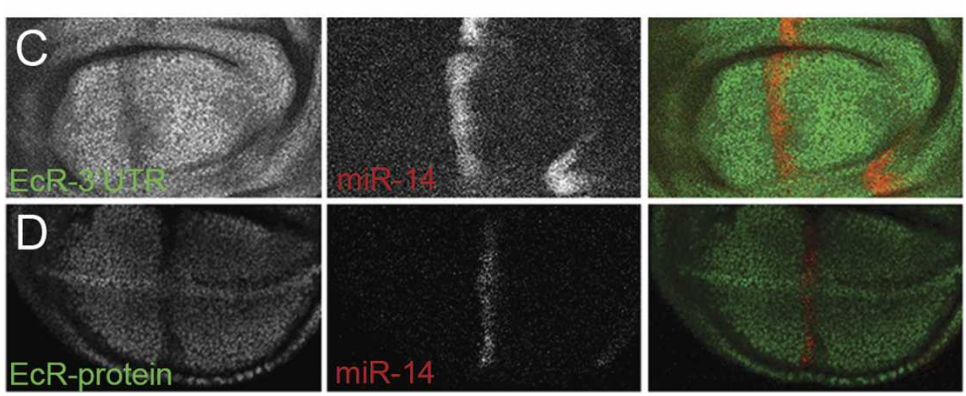

Figure 1. Regulation of EcR by miR-14. (A) Schematic representation of the 3' UTRs of three EcR isoforms. Potential miR-14 target sites conserved in Drosophila pseudoobscura are shown. Blue denotes a site matching only the miRNA "seed" sequence (Brennecke et al. 2005). Green denotes sites with additional complementarity (see Supplementary Fig. S1 for details). (B) Gal4-directed UAS-miR-14 expression inhibited luciferase activity from a reporter containing the EcR 3' UTR, but not from a control 3' UTR luciferase reporter in S2 cells. EcR-mut indicates the EcR 3' UTR luciferase reporter construct in which the seed regions of three conserved miR-14 sites were mutated (see Supplementary Fig. S1 for details). Error bars indicate standard deviation. (C) Portion of a wing imaginal disc from a transgenic fly expressing a GFP reporter containing the $3^{\prime}$ UTR of EcR under control of the tubulin promoter. In control flies the reporter is expressed uniformly in all cells of the wing disc (not shown). Coexpression of UAS-DSred-miR-14 using patched-Gal4 (middle panel, red in merged image) down-regulated reporter levels (left panel, green in merged image). (D) Endogenous EcR protein expression in the wing imaginal disc was down-regulated by overexpression of UAS-DSred-miR-14 using patched-Gal4. EcR has a uniform pattern of expression in the wing disc in the absence of the exogenous miR-14 (not shown).

higher in the miR-14 mutant (Fig. 2D), showing that EcR activity is elevated in miR-14 mutant animals. Differences in the dynamics of their regulation presumably reflect the influence of factors other than EcR.

miR-14 mutants exhibit a variety of defects, including reduced survival to adulthood, reduced adult life span, elevated apoptosis, increased stress response, and abnormal fat metabolism (Xu et al. 2003). To test whether these phenotypes are caused by increased EcR activity, due to loss of miR-14-mediated repression, we removed one functional copy of the $E c R$ gene in the miR-14 mutant background. Reducing $E c R$ gene activity in this way improved the survival to adulthood of homozygous $m i R$ 14 mutants to near normal levels (Fig. 3A). Reduced EcR levels also restored the adult life span of the surviving miR-14 mutant flies to normal (Fig. 3B). Conversely, elevated expression of EcR in miR-14-expressing cells using miR-14-Gal4 reduced survival to adulthood and shortened the life span of adult flies, comparable with the miR-14 mutant phenotypes (Supplementary Fig. S2). Thus elevated EcR in miR-14-expressing cells is sufficient to explain these aspects of the $m i R-14$ mutant phenotype. Reduction of EcR levels did not prevent fat accumulation in the $m i R-14$ mutant, nor did elevated expression of EcR under miR-14 Gal4 control cause fat accumulation, although this defect could be suppressed by restoring miR-14 expression under armadillo Gal4

control (Supplementary Fig. S3). Manipulation of EcR levels also failed to modulate the apoptosis phenotypes of the miR-14 mutant (data not shown).

In addition to the previously described $m i R-14$ mutant phenotypes, we found that anterior spiracle eversion during the larval-pupal transition was defective in miR-14 mutant pupae, suggesting a defect in metamorphosis (Fig. 3C, arrowhead, quantified in D). This phenotype was suppressed almost completely by removing one copy of the EcR gene in the miR-14 mutant background (Fig. 3D), providing genetic evidence that the defect in spiracle metamorphosis is caused by overproduction of EcR in the miR-14 mutant. Overexpression of EcR under miR-14 Gal4 control caused a comparable phenotype in otherwise wild-type animals (Supplementary Fig. S2). The poor survival, reduced adult life span, and metamorphosis defects of miR-14 mutants can therefore be attributed to elevated expression of EcR. Misregulation of other miR-14 targets is likely responsible for the fat accumulation and apoptosis phenotypes.

The observation that miR-14 mutants produce a defect during metamorphosis, due to excess EcR activity, prompted us to examine their regulatory relationship more closely. Previous reports have suggested a direct role for Ecdysone signaling in increasing the levels of EcR during these stages (Koelle et al. 1991; Talbot et al. 1993), but the mechanism by which this regulation takes place has not been addressed. A priori it could be mediated by elevated EcR transcription or by a posttranscriptional effect of miR-14 on EcR levels.

We first compared the level of mature EcR mRNA and the unprocessed EcR primary transcript by quantitative RT-PCR and found that both increased during the early larval-to-pupal transition (Fig. 4A, left). Transcript levels remained high during the mid-pupal stage (P30) and began to decrease during later pupal stages (Fig. 4A, right; see also Riddiford 1993; Thummel 2001). To ask if these changes are due to ecdysone signaling, third instar larval fat bodies were explanted in culture and treated with ecdysone. EcR mRNA and primary transcript levels were elevated in ecdysone-treated samples compared with mocktreated controls (Fig. 4B). Given that ecdysone-mediated transcriptional regulation of many genes during metamorphosis is known to be independent of EcR (Li and White 2003; Beckstead et al. 2005), we asked whether the effects on EcR transcription are EcR dependent. To do so, we made use of RNA interference (RNAi) to knock down levels of the mature EcR mRNA in the cytoplasm. As RNAi does not directly affect the nuclear primary transcript, we used its level to assess EcR transcription. The ability of ecdysone treatment to increase EcR transcript levels was lost in the RNAi-treated samples (Fig. 4B, right). Comparable results were obtained in S2 cells treated with double-stranded RNA (dsRNA) to target EcR or GFP as a control (Fig. 4C). These findings indicate that ecdysone acts via EcR to induce EcR transcription, producing a transcriptional autoregulatory loop that may contribute to ecdysone-induced sensitization in vivo.

Next, we asked what role miR-14 might play in this process. As noted above, genetic evidence indicates that 
A

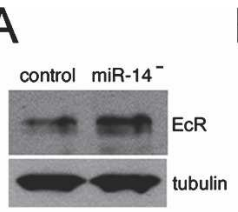

B

D
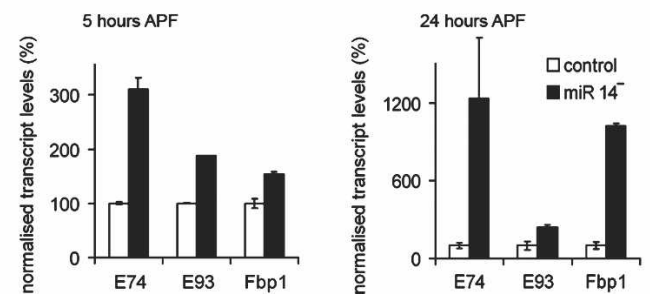

Figure 2. Up-regulation of EcR in miR-14 mutants. (A) Immunoblot of lysates from control $W^{1118}$ and homozygous miR-14 mutant pupae at $24 \mathrm{~h}$ APF probed with an antibody that recognizes all the three isoforms of EcR protein. The blot was reprobed with antitubulin as a loading control. (B) GFP levels in lysates from pupae at $24 \mathrm{~h}$ APF carrying the tubulin promoter-driven EcR-3' UTR GFP reporter transgene in control and miR-14 mutant backgrounds. The blot was reprobed with anti-Kinesin as a loading control. $(C)$ Immunoblot of lysates from pupae at $24 \mathrm{~h}$ APF carrying the Ec-RE:lacZ reporter transgene, in which lac $Z$ expression is under control of the EcR. Probed with anti- $\beta$-galactosidase to monitor EcR activity. The blot was reprobed with anti-Kinesin as a loading control. $(D)$ Transcript levels of Ecdysone-responsive gene levels measured by quantitative real-time PCR from RNA extracted from control and $m i R$ 14 mutant pupae at 5 and $24 \mathrm{~h} \mathrm{APF}$. Error bars show standard deviation from three or more independent biological replicates.

miR-14 normally serves to limit EcR activity in vivo. Interestingly, the transient ecdysone-induced increase in EcR levels during the larval-to-pupal transition is mirrored by a transient decrease in the level of the mature miR-14 miRNA (Fig. 5A; see also Sempere et al. 2003). As some miRNAs induced during pupariation are thought to be independent of Ecdysone signaling or EcR function (Bashirullah et al. 2003), it was important to find out whether miR-14 is regulated by Ecdysone signaling via EcR in vivo. Ecdysone treatment reduced the level of the miR-14 primary transcript in fat body tissue and in S2 cells, but was unable to do so in the samples expressing the UAS-RNAi transgene or treated with dsRNA to knock down mature EcR transcript (Fig. 5B,C). Thus ecdysone-induced down-regulation of miR-14 is mediated by EcR both in vivo and in vitro. In this context it is interesting that EcR mRNA levels were found to be higher in miR-14 mutants (Fig. 5D). This presumably reflects increased EcR autoregulation, as the inhibitory effect of the miRNA is reduced.

These experiments provide evidence that Ecdysone signaling reciprocally regulates transcription of the miR-14 and $E c R$ genes. Thus ecdysone acts in two ways to induce EcR activity: first by promoting EcR transcriptional autoregulation and second by alleviating miR-14-mediated repression of EcR activity (Fig. 5E). Prior to an ecdysone pulse the balance between EcR autoinduction and the mutually repressive interaction between EcR and miR-14 will keep a stable low level of EcR activity. On ecdysone stimulation, the balance shifts to a higher level of EcR activ-
A

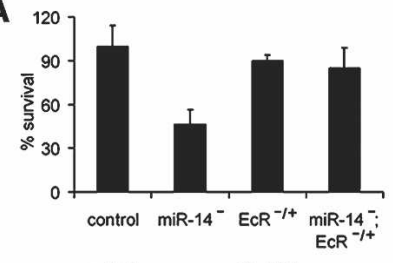

B

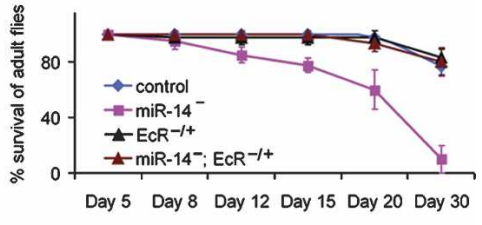

C

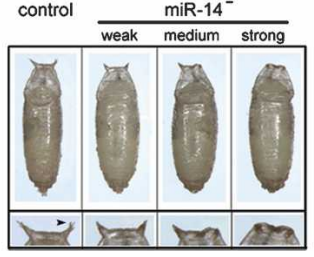

D

\begin{tabular}{|l|c|c|c|c|}
\hline Genotype & $n$ & medium & strong & total \\
\hline w1118 & 329 & $3(0.9)$ & $3(0.9)$ & $6(1.8)$ \\
miR-14 & 242 & $19(7.8)$ & $33(13.6)$ & $52(21.4)$ \\
miR-14 $^{-}$, EcR $^{-1+}$ & 220 & $6(2.7)$ & $3(1.3)$ & $9(4.1)$ \\
\hline
\end{tabular}

Figure 3. Reduction of EcR dosage rescues mutant phenotypes. (A) Histogram showing survival to adulthood of homozygous miR-14 mutant versus control $W^{1118}$ animals. Error bars show standard deviation from at least three independent biological replicates. Survival of $m i R-14$ mutants was significantly different than control flies (Student's $t$-test: $P<0.01$ ). Removing one copy of the EcR $\left(E c R^{V 559 f s} /+\right)$ gene rescued this phenotype and restored survival to the level of the control flies $\left(t\right.$-test $m i R-14 ; E c R^{V 559 f s} /+$ vs. control: $P=0.25$; for $m i R-14$, $E c R^{V 559 f s} /+$ vs. $m i R-14$ : $\left.P<0.01\right)$. (B) Graph showing the life span of miR-14 mutant adults versus control $w^{1118}$ flies. Flies lacking one copy of EcR $\left(E c R^{V 559 f s} /+\right)$ increased life span of the miR-14 mutant. Error bars are as in $A .(C)$ miR-14 mutant pupae show anterior spiracle eversion defects; the bottom panel shows the anterior spiracles (arrowhead) at higher magnification. (Weak) Reduction of spiracle length; (medium and strong) failure to evert one or both spiracles. $(D)$ Quantification of the anterior spiracle phenotype; " $n$ " denotes the number of pupae counted for each genotype; in parenthesis is the percentage of pupae showing the phenotype. 
A

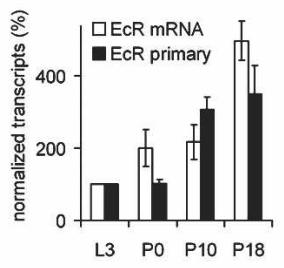

B

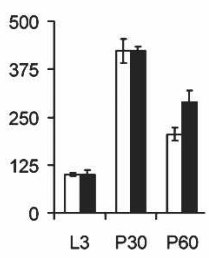

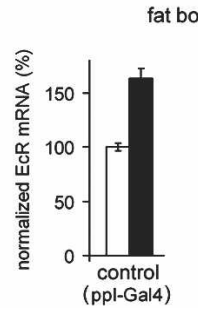

C

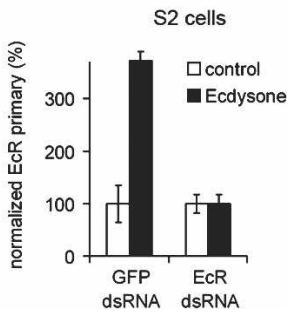

Figure 4. Ecdysone acts via EcR to positively regulate EcR transcription. $(A)$ EcR mature mRNA levels and EcR primary transcript levels measured by quantitative RT-PCR. mRNA levels were measured using exonic primers that span a spliced intron. The primary transcript was measured using intronic primers. (L3) Third larval instar; (P0) white prepupae; (P10, P18, P30, and P60) 10, 18, 30-36 and 56-64 h APF, respectively. Error bars in all panels represent standard deviation of at least three independent biological replicates. $(B)$ EcR mature mRNA levels (left) and EcR primary transcript levels (right) measured by quantitative RT-PCR. Third instar larval fat bodies were explanted into culture and treated for $6 \mathrm{~h}$ with $10 \mu \mathrm{M} 20-\mathrm{OH}$ ecdysone (black bars) or with the ethanol vehicle as a control (white bars). Control animals carried one copy of the pumpless-Gal4 driver (ppl-Gal4), which is expressed in the fat body. ppl-Gal4 was used to direct a UAS-RNAi transgene to knock down cytoplasmic EcR mRNA by RNAi. (C) EcR primary transcript levels determined by quantitative RT-PCR on RNA extracted from S2 cells treated with dsRNA to EcR or to GFP, and treated with $20 \mu \mathrm{M}$ 20-OH Ecdysone or mock-treated with ethanol as a control.

helix (bHLH) transcription factors form a positive regulatory loop with the zinc-finger transcription factor Senseless (Nolo et al. 2000). miR-9a sets a threshold of activity that must be exceeded for the loop to activate by limiting Senseless expression. When coupled with a positive autoregulatory circuit, miRNAs can provide an effective means by which to set thresholds and limit noise-induced errors to ensure robustness in development (Cohen et al. 2006).

\section{Materials and methods}

Reagents and fly strains

The miR-14 deletion mutant was kindly provided by Bruce Hay (Xu et al. 2003). The UAS-EcR-RNAi line was kindly provided by Pierre Leopold
(Colombani et al. 2005). The patched-Gal4, armadillo-Gal4, UAS-EcR$B 1, U A S-G F P, E c R^{V 559 f s}$, and $E c-R E$ :lacZ reporter strains were from the Bloomington Stock Center and are described in FlyBase (http://flybase. bio.indiana.edu). UAS-miR14 and tub-miR-14 constructs were generated by cloning a 500-base-pair (bp) genomic fragment containing the miR-14 hairpin into pUAST vector containing the dsRed coding sequence and into a tubulin promoter vector, respectively. The EcR-3' UTR GFP reporter strain was generated by cloning the 1.8 -Kb 3' UTR from EcR after GFP, under control of a tubulin promoter (as described in Brennecke et al. 2003). The EcR-3' UTR luciferase reporters were generated similarly in an S2 cell expression vector with the firefly luciferase. EcR-3' UTR reporters with miR-14 sites mutated were generated using PCR amplification by specific primers designed to incorporate the mutations. Control 3' UTR reporters contain the 3' UTRs of GFP and luciferase in the respective cases. The miR-14 P-Gal4 insertion line NP-4722 was from GET-DB, DGRC, Kyoto. Antibodies to EcR protein were from the
A

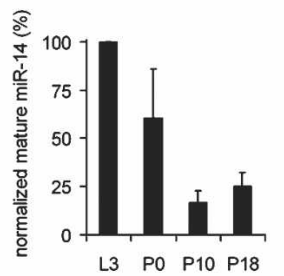

D

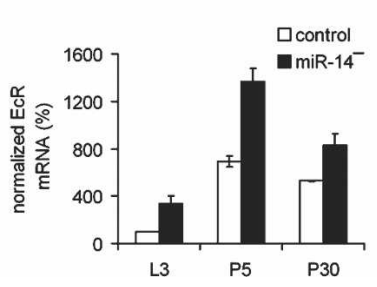

B

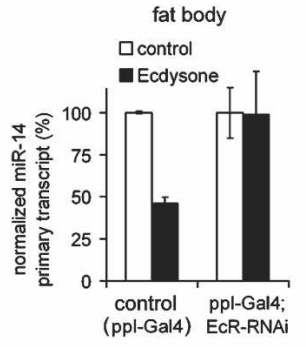

C

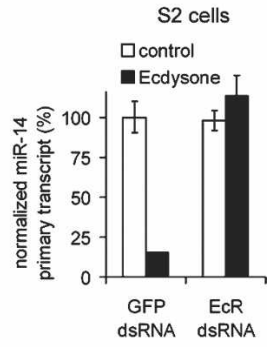

E

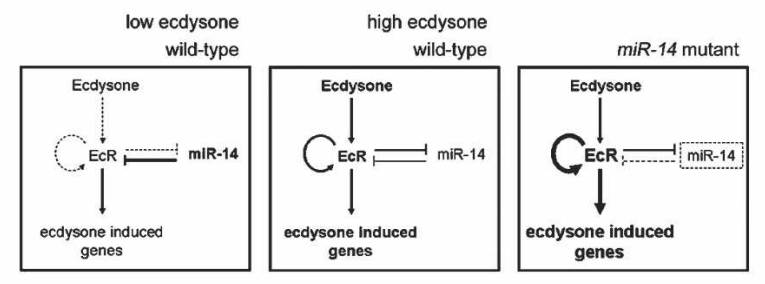

Figure 5. Ecdysone acts via miR-14 to alleviate repression of EcR. (A) Measurement of mature miR-14 miRNA levels by quantitative RT-PCR from total RNA extracted from control animals at the indicated stages. Error bars in $A-D$ represent standard deviation of at least three independent biological replicates. $(B, C)$ miR-14 primary transcript levels measured by quantitative RT-PCR from total RNA from fat body tissue $(B)$ or S2 cells $(C)$ treated with dsRNA to EcR or to GFP. Samples in $B$ were treated for $6 \mathrm{~h}$ with $10 \mu \mathrm{M} 20$-OH ecdysone or mock-treated with ethanol; those in $C$ were treated with $20 \mu \mathrm{M} 20-\mathrm{OH}$ ecdysone for $6 \mathrm{~h}$. $(D)$ EcR mRNA levels measured by quantitative RT-PCR in RNA from control or miR-14 mutant animals at the indicated stages. (E) Model showing regulation of steroid hormone signaling by EcR and miR-14. (Left panel) Low level of EcR activity under conditions of low ecdysone stimulation. Repression by miR-14 dominates. (Middle panel) Effects of sustained activation by ecdysone overcomes miR-14-mediated repression and allows strong autoactivation of EcR. (Right panel) In the absence of miR-14, EcR autoactivation is not limited and can reach higher levels. 
Developmental Studies Hybridoma Bank. Anti- $\beta$-Gal was from Promega, anti-GFP was from Torrey Pines Biolabs, and anti-Kinesin was from $\mathrm{Cy}$ toskeleton. The 20-Hydroxyecdysone was purchased from Sigma-Aldrich and was maintained as a $10 \mathrm{mM}$ stock in ethanol.

Mutant phenotype analysis and staged samples for total

RNA extraction

For phenotype analysis, flies were raised under controlled conditions. Embryos were collected after $6 \mathrm{~h}$ of egg laying on apple-agar plates in embryo collection cages, and 50 first instar larvae were collected simultaneously from all the genotypes (24-30 h after egg laying) into fresh vials. The number of pupae with anterior spiracle eversion defects was counted from the vials for the various genotypes. For the survival assays, the number of adult flies was counted in 10 vials of each genotype after $12 \mathrm{~d}$. Male and female flies emerging were collected separately in batches of 10 each and placed in fresh vials. Flies were transferred to fresh vials on alternate days and the number of surviving flies was counted to measure adult life span. The Triglyceride assay was performed as described in Teleman et al. (2006). Total RNA was extracted using the Trizol method from late third instar larvae, $\sim 12 \mathrm{~h}$ before puparium formation (L3), from prepupae (P0) $0 \mathrm{~h} \mathrm{APF}$, from pupae at 5 (P5), 10 (P10), 18 (P18), and 30-36 h APF (P30), and from late pupae 58-64 h APF (P60).

\section{Immunoblotting}

Protein extracts were made from flies or pupae raised as described above by crushing them in SDS sample buffer and boiling the samples for $5 \mathrm{~min}$. Samples were resolved in $8 \%$ or $12 \%$ SDS-PAGE gels, transferred to nitrocellulose membranes, incubated with primary antibodies diluted in $5 \%$ fat-free milk, and visualized using secondary antibodies conjugated with HRP followed by ECL reagent according to the manufacturer's instructions (Perkin-Elmer). Primary antibody dilutions were 1:100 for anti-EcR common antibody, 1:5000 for anti- $\beta \mathrm{Gal}$, and 1:10,000 for antiTubulin and anti-Kinesin. Membranes were stripped and reprobed with anti-Tubulin or anti-Kinesin to compare loading.

Ecdysone treatment on fat body and S2 cells

Fat bodies from third instar larvae were dissected into cold Drosophila SFM and were treated with $10 \mu \mathrm{M} 20-\mathrm{OH}$ ecdysone or with an equal volume of ethanol (Karim and Thummel 1992). The samples were incubated for $6 \mathrm{~h}$ at $25^{\circ} \mathrm{C}$. S2 cells were treated with $12 \mu \mathrm{g}$ of dsRNA generated against GFP or a region common to all EcR isoforms (Hipfner and Cohen 2003; Colombani et al. 2005) for $72 \mathrm{~h}$. The cells were then treated with $20 \mu \mathrm{M} 20-\mathrm{OH}$ ecdysone or ethanol for $6 \mathrm{~h}$ (Karim and Thummel 1992).

Luciferase reporter assays

S2 cells were transfected in 24-well plates with $250 \mathrm{ng}$ of tubulin-miR14 plasmid DNA or the empty tubulin vector, $25 \mathrm{ng}$ of firefly luciferase DNA or EcR-3' UTR or mutant luciferase reporter DNA, and $25 \mathrm{ng}$ of Renilla luciferase DNA as a transfection control. Dual luciferase assays were performed $60 \mathrm{~h}$ post-transfection according to the manufacturer's protocol (Promega).

Antibody staining and immunofluorescence

Wing imaginal discs from nonwandering third instar larvae were dissected and incubated with anti-EcR common antibody (AG 10.2 monoclonal antibody 1:10 dilution) after fixation in $4 \%$ formaldehyde/PBS. Anti-mouse IgG-conjugated with FITC (Jackson Laboratory) was used to fluorescently label the samples and was visualized by confocal microscopy.

Quantitative PCR ( $q P C R$ ) for primary and mature miR-14 For the miR-14 primary qPCR, gene-specific primers were designed $\sim 200$ bp upstream of the miRNA stem-loop that was used for the first strand synthesis. qPCR analysis was carried out using primer pairs designed within $150 \mathrm{bp}$ upstream of the gene-specific primer-binding site. For the mature miRNA analysis, the primer sets designed to amplify mature miR-14 were obtained from Applied Biosystems. The qPCR was done according to the manufacturer's protocol. Products were amplified from 10 ng of total RNA with the "TaqMan MicroRNA Assay," Quantitative PCR machine, and software from Applied Biosystems. The miR-14 levels was calculated relative to either of the two references, $5 \mathrm{~s}$ rRNA or U6 snRNA.
qPCR for various gene transcripts

Total RNA extracted from the samples was treated with DNase-1 to eliminate genomic DNA contamination. The reverse transcription reaction to synthesyze the first strand was done by using oligo-dT primers and SuperScript RT-III (Invitrogen). The samples were RNase-H-treated after the RT reaction and then used for the qPCR. Primers were designed using Oligo.6 primer analysis software. Primers for EcR were designed against a region common to all five transcripts. Primers to analyze transcriptional regulation of EcR were designed against intronic regions common to all the five-EcR pre-mRNA forms, as follows: miR-GSP1, 5'GCTGGCTGATAAATAATGAA-3'; miR-GSP, 5'-GTTGGTGCTGGC TGATAAAT-3'; miR-1, 5'-CACCCAAATCCAAAGAGAATGA-3'; miR-2, 5'-CGAAACCACGGCAACAGACTT-3'; miR-3, 5'-GCCGAATCAATTAC GCAACTT-3'; miR-4, 5'-TTCCGATAATGAAAAGCACACA-3'; EcR-1, 5'-TAACGGCCAACTGATTGTACG-3'; EcR-2, 5'-GCGGCCAAGACT TTGTTAAGA-3'; EcR-3, 5'-TCATAGCCATCCTGGTACCAAA-3'; EcR-4, 5'-TGACCTTATGACATGCGAGCC-3'; EcR-5， 5'-ATGAGCGGAGTC TAAAAAAATCG-3'; EcR-6, 5'-GGAGCCGAACAGAACAATAAGAG 3'; EcR-7, 5'-GAAATCACTGGGTACAACCGAA-3'; EcR-8, 5'-GAATAT TGAGTCCGAGCTGTGG-3'; EcR-9, 5'-TTCCAAACACTCAGCCCT CAAG-3'; EcR-10, 5'-GAGGGAACACAGATAAGTAGGCG-3'; E74-1, 5' GAATCCGTAGCCTCCGACTGT-3'; E74-2, 5'-AGGAGGGAGAGTGG TGGTGTT-3'; E74-3, 5'-GATGGGCAGCAGGCTAAGACT-3'; E74-4, 5'CTTCACCTCTTGCCTCGTCTTT-3'; E93-1, 5'-GCGATGTGAAGTCC GTCAGA-3'; E93-2, 5'-TTTCCGGGCATCTAGCTTGT-3'; E93-3, 5'CAAACCATCGCCGAACTCAT-3'; E93-4, 5'-TTCCGGGCATCTAGCT TGTT-3'; Fbp1-1， 5'-AACGGTGGGCAGGTTATGAG-3'; Fbp1-2, 5'TATTCGCATGGGTCGTGTCC-3'; Fbp1-3, 5'-GCTGGGAATTGATTC GGATTT-3'; Fbp1-4, 5'-CAGCTGGTCGCACGTCTTAAC-3'.

\section{dsRNA primers}

DsRNA primers were as follows: GFP IR1, 5'-AGGAGCTGTTCACC GGGG-3'; GFP IR2, 5'-TCGCGCTTCTCGTTGGGG-3'; EcR IR1, 5'AAGAATTCGGTACCAGGATGGCTATGAG-3'; EcR IR2, 5' '-TTAG ATCTCCTCGAGGAACTTG-3'.

\section{Acknowledgments}

We thank Bruce Hay and Pierre Leopold for flies, Ann-Mari Voie for preparing transgenic flies, Jens Stolte for the mature microRNA qPCRs, and Ville Hietakangas, Sebastien Szuplewski, and Aurelio Teleman for comments on the manuscript. J.V. was a fellow of the Alexander von Humboldt foundation and was supported by an International research fellowship from Deutsche Forschungsgemeinschaft (DFG).

\section{References}

Abbott, A.L., Alvarez-Saavedra, E., Miska, E.A., Lau, N.C., Bartel, D.P., Horvitz, H.R., and Ambros, V. 2005. The let-7 MicroRNA family members mir-48, mir-84, and mir-241 function together to regulate developmental timing in Caenorhabditis elegans. Dev. Cell 9: 403414.

Bashirullah, A., Pasquinelli, A.E., Kiger, A.A., Perrimon, N., Ruvkun, G., and Thummel, C.S. 2003. Coordinate regulation of small temporal RNAs at the onset of Drosophila metamorphosis. Dev. Biol. 259: 1-8.

Beckstead, R.B., Lam, G., and Thummel, C.S. 2005. The genomic response to 20-hydroxyecdysone at the onset of Drosophila metamorphosis. Genome Biol. 6: R99. doi: 10.1186/gb-2005-6-12-r99.

Brennecke, J., Hipfner, D.R., Stark, A., Russell, R.B., and Cohen, S.M. 2003. bantam encodes a developmentally regulated microRNA that controls cell proliferation and regulates the proapoptotic gene hid in Drosophila. Cell 113: 25-36.

Brennecke, J., Stark, A., Russell, R.B., and Cohen, S.M. 2005. Principles of microRNA-target recognition. PLoS Biol. 3: e85. doi: 10.1371/ journal.pbio.0030085.

Bushati, N. and Cohen, S.M. 2007. microRNA Functions. Annu. Rev. Cell Dev. Biol. doi: 10.1146/annurev.cellbio.23.090506.123406.

Carthew, R.W. 2006. Gene regulation by microRNAs. Curr. Opin. Genet. Dev. 16: 203-208.

Cohen, S.M., Brennecke, J., and Stark, A. 2006. Denoising feedback loops by thresholding-A new role for microRNAs. Genes \& Dev. 20: 2769-2772.

Colombani, J., Bianchini, L., Layalle, S., Pondeville, E., Dauphin-Ville- 
mant, C., Antoniewski, C., Carre, C., Noselli, S., and Leopold, P. 2005. Antagonistic actions of ecdysone and insulins determine final size in Drosophila. Science 310: 667-670.

Enright, A.J., John, B., Gaul, U., Tuschl, T., Sander, C., and Marks, D.S 2003. MicroRNA targets in Drosophila. Genome Biol. 5: R1. doi: 10.1186/gb-2003-5-1-r1.

Grosshans, H., Johnson, T., Reinert, K.L., Gerstein, M., and Slack, F.J. 2005. The temporal patterning microRNA let-7 regulates several transcription factors at the larval to adult transition in C. elegans. Dev. Cell 8: 321-330.

Hipfner, D.R. and Cohen, S.M. 2003. The Drosophila sterile-20 kinase slik controls cell proliferation and apoptosis during imaginal disc development. PLOS Biol. 1: E35. doi: 10.1371/journal.pbio.0000035.

Karim, F.D. and Thummel, C.S. 1992. Temporal coordination of regulatory gene expression by the steroid hormone ecdysone. EMBO J. 11: 4083-4093.

Kloosterman, W.P. and Plasterk, R.H. 2006. The diverse functions of microRNAs in animal development and disease. Dev. Cell 11: 441450.

Koelle, M.R., Talbot, W.S., Segraves, W.A., Bender, M.T., Cherbas, P., and Hogness, D.S. 1991. The Drosophila EcR gene encodes an ecdysone receptor, a new member of the steroid receptor superfamily. Cell 67: 59-77.

Lee, R.C., Feinbaum, R.L., and Ambros, V. 1993. The C. elegans heterochronic gene lin-4 encodes small RNAs with antisense complementarity to lin-14. Cell 75: 843-854.

Li, T.R. and White, K.P. 2003. Tissue-specific gene expression and ecdysone-regulated genomic networks in Drosophila. Dev. Cell 5: 59-72.

Li, M., Jones-Rhoades, M.W., Lau, N.C., Bartel, D.P., and Rougvie, A.E. 2005. Regulatory mutations of mir-48, a C. elegans let-7 family $\mathrm{Mi}$ croRNA, cause developmental timing defects. Dev. Cell 9: 415-422.

Li, Y., Wang, F., Lee, J.A., and Gao, F.B. 2006. MicroRNA-9a ensures the precise specification of sensory organ precursors in Drosophila. Genes \& Dev. 20: 2793-2805.

Massirer, K.B. and Pasquinelli, A.E. 2006. The evolving role of microRNAs in animal gene expression. Bioessays 28: 449-452.

Nolo, R., Abbott, L.A., and Bellen, H.J. 2000. Senseless, a Zn finger transcription factor, is necessary and sufficient for sensory organ development in Drosophila. Cell 102: 349-362.

Olsen, P.H. and Ambros, V. 1999. The lin-4 regulatory RNA controls developmental timing in Caenorhabditis elegans by blocking LIN-14 protein synthesis after the initiation of translation. Dev. Biol. 216: 671-680.

Pasquinelli, A.E., Reinhart, B.J., Slack, F., Martindale, M.Q., Kuroda, M.I., Maller, B., Hayward, D.C., Ball, E.E., Degnan, B., Muller, P., et al. 2000. Conservation of the sequence and temporal expression of let-7 heterochronic regulatory RNA. Nature 408: 86-89.

Reinhart, B.J., Slack, F.J., Basson, M., Pasquinelli, A.E., Bettinger, J.C., Rougvie, A.E., Horvitz, H.R., and Ruvkun, G. 2000. The 21-nucleotide let-7 RNA regulates developmental timing in Caenorhabditis elegans. Nature 403: 901-906.

Riddiford, L.M. 1993. Hormone receptors and the regulation of insect metamorphosis. Receptor 3: 203-209.

Sempere, L.F., Sokol, N.S., Dubrovsky, E.B., Berger, E.M., and Ambros, V. 2003. Temporal regulation of microRNA expression in Drosophila melanogaster mediated by hormonal signals and broad-Complex gene activity. Dev. Biol. 259: 9-18.

Stark, A., Brennecke, J., Russell, R.B., and Cohen, S.M. 2003. Identification of Drosophila microRNA targets. PLoS Biol. 1: E60. doi: 10.1371/ journal.pbio.0000060.

Talbot, W.S., Swyryd, E.A., and Hogness, D.S. 1993. Drosophila tissues with different metamorphic responses to ecdysone express different ecdysone receptor isoforms. Cell 73: 1323-1337.

Teleman, A.A., Maitra, S., and Cohen, S.M. 2006. Drosophila lacking microRNA miR-278 are defective in energy homeostasis. Genes \& Dev. 20: 417-422.

Thummel, C.S. 1996. Files on steroids-Drosophila metamorphosis and the mechanisms of steroid hormone action. Trends Genet. 12: $306-$ 310 .

Thummel, C.S. 2001. Molecular mechanisms of developmental timing in C. elegans and Drosophila. Dev. Cell 1: 453-465.

Valencia-Sanchez, M.A., Liu, J., Hannon, G.J., and Parker, R. 2006. Control of translation and mRNA degradation by miRNAs and siRNAs.
Genes \& Dev. 20: 515-524.

White, K.P., Hurban, P., Watanabe, T., and Hogness, D.S. 1997. Coordination of Drosophila metamorphosis by two ecdysone-induced nuclear receptors. Science 276: 114-117.

Wightman, B., Ha, I., and Ruvkun, G. 1993. Posttranscriptional regulation of the heterochronic gene lin-14 by lin- 4 mediates temporal pattern formation in C. elegans. Cell 75: 855-862.

Xu, P., Vernooy, S.Y., Guo, M., and Hay, B.A. 2003. The Drosophila microRNA Mir-14 suppresses cell death and is required for normal fat metabolism. Curr. Biol. 13: 790-795. 


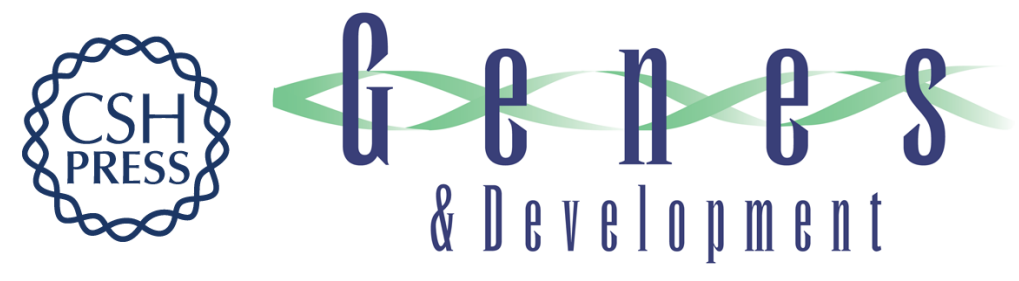

\section{microRNA miR-14 acts to modulate a positive autoregulatory loop controlling steroid hormone signaling in Drosophila}

Jishy Varghese and Stephen M. Cohen

Genes Dev. 2007, 21: originally published online August 30, 2007

Access the most recent version at doi:10.1101/gad.439807

\section{Supplemental http://genesdev.cshlp.org/content/suppl/2007/08/30/gad.439807.DC1 \\ Material}

Related Content

References

\section{License}

Email Alerting

Service
This article cites 34 articles, 6 of which can be accessed free at:

http://genesdev.cshlp.org/content/21/18/2277.full.html\#ref-list-1

Articles cited in:

http://genesdev.cshlp.org/content/21/18/2277.full.html\#related-urls

Control of a Metamorphic Switch

L. Bryan Ray

Sci. STKE September , 2007 2007: tw342

Receive free email alerts when new articles cite this article - sign up in the box at the top right corner of the article or click here.

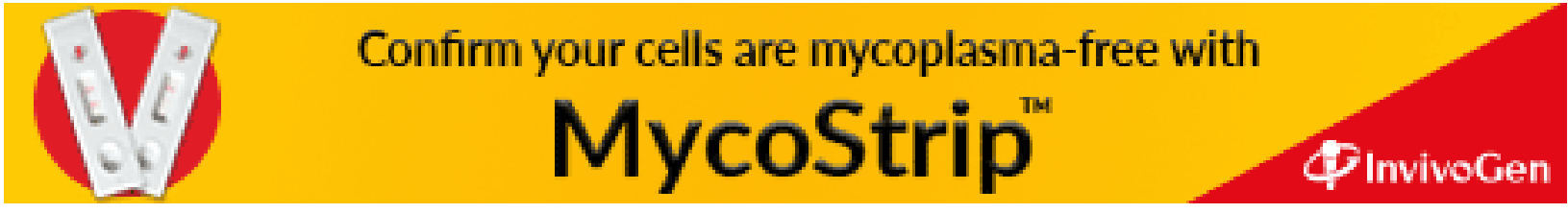

